\title{
Avaliação da propagação de sinais de radiofrequência para tecnologia Zigbee em granja de frango de corte
}

\author{
Eduardo F. N unes $^{1}$, Nelson L. Cappelli ${ }^{1} \&$ Claudio K. U mezu ${ }^{1}$
}

\section{RESUMO}

Visou-se, neste trabalho, realizar a predição de sinais de radiofrequência para a aplicação de Rede de Sensores Sem Fio (RSSF) de monitoramento e controle em uma granja de frango de corte. A tecnologia de comunicação sem fio utilizada foi a Zigbee, com o padrão IEEE 802.15.4 compondo a camada física e de enlace. D esenvolveu-se, para automatizar a leitura de potências, um aplicativo em linguagem Delphi e, para a predição de sinais de RF (Radiofrequência), utilizou-se o modelo Shadowing adaptado. Todos os testes foram realizados em granja comercial, no M unicípio de Tuiuti, estado de São Paulo, Brasil. Os resultados obtidos demonstraram boas condições de transmissão até $131 \mathrm{~m}$, indicando que a tecnologia Zigbee é adequada, segundo a perspectiva de propagação de sinais, para a implementação de RSSF em granjas de frango de corte.

Palavras-chave: rede de sensores sem fio, ieee 802.15.4, comunicação sem fio, modelo de shadowing

\section{Evaluation of radiofrequency signals for Zigbee technology in poultry housing}

\section{ABSTRACT}

This study aimed to conduct an evaluation for the implementation of W ireless Sensors N etw ork (W SN) of tracking and control in a poultry farm. The wireless technology used was Zigbee, with the IEEE 802.15.4 standard forming the physical layer and link layer. To automate the power measurement a DELPH I language software was developed and, for the prediction of RF signals, an adjusted model of the shadowing was used. All tests were performed in a commercial broiler farm, in the city of Tuiuti in the State of São Paulo, Brazil. The results of this study indicate that Zigbee technology is appropriate, from the perspective of signal propagation, to implement WSN in poultry housing.

Key words: wireless sensor network, ieee 802.15.4, wireless communication, shadowing model 


\section{INTRODUÇÃO}

Na avicultura de corte é grande a importância das condições ambientais a que as aves são submetidas. A concentração de animais e os índices de produtividade necessários para que o empreendimento seja viável, fazem com que variações ambientais influenciem, em muito, no resultado final para o produtor.

Condições ambientais adversas contribuem negativamente em índices como a conversão alimentar e, segundo Sevegnani (2005), o estresse calórico tem impacto na conversão alimentar com efeitos economicamente significantes. $\mathrm{O}$ autor enfatiza, também, que o próprio desenvolvimento das aves aquece o ambiente pois a temperatura corporal dos frangos aumenta com o consumo de alimentos, com a taxa de crescimento e a eficiência alimentar, ou seja, diversos fatores influenciam nas condições ambientais das granjas.

De acordo com Moura (2001), na avicultura de corte, se a ave estiver submetida a uma temperatura efetiva adequada, sem nenhum desperdício de energia, tanto para compensar o frio como para acionar seu sistema de refrigeração, pode-se chegar a ótimos índices de produtividade. $\mathrm{O}$ autor enfatiza, ainda, que a temperatura efetiva não pode ser compreendida unicamente com a temperatura ambiental, pois ela depende da combinação da temperatura de bulbo seco, umidade relativa, radiação solar e velocidade do vento.

Segundo Tinôco (2001), a crescente demanda por produtividade e a implantação dos sistemas de integrados de produção trouxeram à tona questões relativas à ambiência. Hoje, é imprescindível que cada empresa busque e incentive novas técnicas de manejo, estruturas arquitetônicas e outras tecnologias para o condicionamento térmico apropriado nas granjas de seus integrados.

Lima (2005) aponta o crescente incremento tecnológico na produção de frangos como responsável pelos altos índices de produtividade hoje obtidos. Na busca de melhores índices zootécnicos, novas técnicas para monitorar e atuar em prol do conforto animal, foram desenvolvidas. O uso de imagens captadas na granja subsidia análises sobre o conforto dos animais. A contagem do uso dos bebedouros e comedouros é realizada por técnicas de processamento de vídeo ou de quadros de imagens consecutivos.

Silva et al. (2005) relatam o desenvolvimento de uma rede de sensores sem fio (RSSF) para monitorar a resposta fisiológica de rebanhos bovinos, em que os sensores são instalados nos animais e uma estação base concentra e processa os dados. Na mesma linha de pesquisa Nadimi et al. (2008) monitoram a presença de bovinos e o tempo de pastagem, através de uma RSSF, na qual apenas $23 \%$ dos animais estavam equipados com nós sensores, obtendo-se uma assertividade de 79,9\% nos resultados finais. Já Green et al. (2009), utilizaram uma rede de sensores sem fio para monitorar as variações de temperatura dentro de uma pilha de silagem, demonstrando assertividade para detectar o grau de decomposição e subsidiar melhorias no processo de conservação.

Entretanto, na grande maioria das pesquisas realizadas com RSSF aplicada na agricultura, o ambiente no qual a rede é instalada não é caracterizado em seus aspectos de propagação de sinais eletromagnéticos, como nos trabalho de Riquelme et al. (2009).

Especificamente para o monitoramento da produção de frango de corte, deve-se levar em conta o ambiente hostil aos dispositivos eletrônicos, como poeira, gases dissolvidos, temperaturas elevadas, umidade e vibrações mecânicas que aceleram a degradação desses equipamentos, além da existência de interferências eletromagnéticas de equipamentos, como motores.

Outra característica significativa levada em consideração é a reduzida dinâmica de variação das grandezas ambientais, como temperatura e umidade relativa, que variam em um espaço de tempo longo, usualmente na casa de minutos; desta forma, a amostragem pode ser espaçada para intervalos de minutos conferindo, a esses dispositivos, um uso de banda espectral reduzido no tempo, e baixo consumo de energia.

A robustez necessária, a demanda de baixa taxa de transmissão e as pequenas distâncias dentro de um galpão, apontam para as soluções de RSSF utilizando-se a tecnologia Zigbee (Wang et al., 2006). Além de contemplar as especificidades de controles e registros usados na produção intensiva de frango de corte, a tecnologia Zigbee/802.15.4 escolhida, pertence à "família" de normativas IEEE 802. Notoriamente, os dispositivos desta "família" dominam o mercado e ganharam escala como, por exemplo, a IEEE $802.3 \mathrm{e}$ a IEEE 802.11, cuja consequência reside nos preços cada vez mais reduzidos e acessíveis.

Zigbee e 802.15.4 (IEEE, 2003) não são sinônimos porém são tecnologias complementares que, juntas, consolidam a pilha de protocolos de comunicação utilizada neste trabalho.

O desenvolvimento desta predição de sinais para tecnologia Zigbee/802.15.4 é fruto da aspiração por, cada vez mais, informações do processo de produção de frango de corte e dos ganhos oriundos de uma gestão eficiente dessas informações e também do uso cada vez maior e consequente consolidação e acessibilidade da tecnologia Zigbee.

Neste contexto, a avaliação da propagação de sinais de radiofrequência para tecnologia Zigbee em granja de frango de corte, se faz necessária para se conhecer os limites da aplicação e subsidiar trabalhos nesta linha de pesquisa.

\section{MATERIAL E MÉTODOS}

Para o desenvolvimento da pesquisa foram utilizados os seguintes materiais:

- Kit de desenvolvimento Zigbee: Modelo Xbee Development Kit, marca MaxStream, com três dispositivos Xbee, dois com antenas externas de $2,1 \mathrm{~dB}$ e um com antena interna (todas com polarização vertical), três interfaces de comunicação serial, sendo duas interfaces RS-232 e uma USB.

- Computador portátil: modelo Aspire 3003LCi, marca Acer, processador AMD Sempron 3000+1,8 GHz, memória de 448 MB e 3 interfaces USB.

- Plataforma de desenvolvimento de software: Modelo Delphi 7.0, marca Borland.

O kit de desenvolvimento com interface de rede do tipo Zigbee/802.15.4, denominado Xbee, da empresa MaxStream, 
possui um microprocessador e todo o suporte para utilização de aplicações que envolvam as camadas Zigbee.

\section{Ferramenta computacional}

Desenvolveu-se, para coletar as medidas de laboratório e de campo, uma ferramenta computacional para acionar o tráfego entre dois dispositivos Xbee (para cada tipo de sensor foi criado um tipo de tráfego), ler a potência de sinal e contabilizar os pacotes perdidos. Entende-se por pacotes perdidos aqueles que são transmitidos porém não chegam ao outro elemento comunicante ou então não podem ser interpretados adequadamente devido a alguma distorção do sinal.

$\mathrm{O}$ algoritmo desenvolvido em linguagem Delphi (Borland Delphi 7) foi baseado em sequências de comandos AT.

Um dos dispositivos Xbee se conecta ao computador portátil e recebe os comandos via porta de comunicação serial USB.

Inicialmente são enviadas strings (de caracteres ASCII) com a finalidade de criar tráfego na rede; no outro extremo da rede o segundo dispositivo Xbee recebe as strings e, através de um loop-back na porta de comunicação RS-232, essas sentenças são enviadas de volta ao dispositivo Xbee conectado ao computador portátil.

Comparando as sentenças enviadas com as sentenças recebidas, pode-se identificar a integridade e perda dos pacotes; a cada pacote recebido se identifica, também, a potência de recepção.

\section{Caracterização da granja}

De início, caracterizou-se a granja nos seus aspectos dimensionais, em uma planta baixa do galpão; a caracterização física subsidiou e complementou a predição de sinais da RSSF na granja. O modelo adotado para a predição foi o Shadowing adaptado (Fanimokun \& Frolik, 2003).

O modelo Shadowing adaptado (Eq. 1) de predição se baseia na correção do expoente da distância, ou seja, para diferentes ambientes existem diferentes expoentes tabelados. No caso de um ambiente de espaço livre este expoente ( $\beta$ ) é igual a dois e o valor de $X$ é representado pelo desvio padrão de uma distribuição gaussiana da atenuação.

$$
\mathrm{L}[\mathrm{dB}]=-10 * \beta * \log (\mathrm{d})+\mathrm{X}[\mathrm{dB}]
$$

Este modelo foi escolhido em virtude da determinação de $\beta$ e de X ser realizada empiricamente no local onde a rede será instalada.

Como a energia do canal foi mensurada instantes antes de cada transmissão e da medida de potência, pode-se obter a relação da potência de recepção pela energia do canal quando este está inoperante, isto é, obtém-se a relação sinal/ruído (SNR).

Partindo-se da constatação de que as medidas foram realizadas com a granja em funcionamento, este modelo consegue incorporar, na equação, as obstruções existentes no ambiente, como equipamentos e estruturas dentro do galpão; inclui, também, as possíveis fontes de interferência que degradam o sinal transmitido, seja aumentando o valor de $\beta$ ou o offset de atenuação; desta forma, as interferências geradas por outras redes sem fio, motores, máquinas e equipamentos eletrônicos, são contabilizadas.
Para se obter as equações que caracterizam o galpão, foram realizadas medidas em duas radiais, com média de 10 pontos por radial e cada ponto com aproximadamente 100 amostras de potência e de energia do canal daquele ponto. A partir das 100 amostras obteve-se a média aritmética da potência recebida e da energia do canal para cada ponto.

Em um gráfico de dispersão da razão sinal-ruído (SNR) pela distância foi extraída uma curva logarítmica aproximada que melhor representasse essa distribuição e desta equação se isolou o coeficiente usado para caracterizar o ambiente $(\beta)$.

Espacialmente, a granja possuía $125 \mathrm{~m}$ de comprimento, $12 \mathrm{~m}$ de largura e pé direito com 3,5 m. Os equipamentos da granja são distribuídos ao longo do galpão, a uma altura de 30 $\mathrm{cm}$ (Figura 1). A estrutura é composta de pilares de concreto e telas metálicas com malha de $4 \mathrm{~cm}$ e, entre cada galpão, a distância é de aproximadamente $10 \mathrm{~m}$.



Figura 1. Vista interior do galpão utilizado nos testes

Ao longo do galpão existiam três linhas de comedouros e cinco linhas de bebedouros tipo nipple, suspensos por cabos de aço. Os comedouros eram automáticos com motor elétrico para movimentar a rosca sem fim; já o sistema dos bebedouros empregava a gravidade para manter a oferta de água; as linhas de bebedouros e comedouros estavam dispostas de maneira intercalada dentro do galpão.

Duas radiais foram traçadas para guiar as medidas de campo: uma longitudinal ao galpão, percorrendo toda a granja (radial 1) e outra, a radial 2 , foi traçada de tal maneira a existir um número maior de obstáculos entre os dois módulos. Entre as duas extremidades existem três pratos comedouros e as quatro linhas de bebedouros. As radiais estão mostradas na Figura 2.



Figura 2. Posicionamento das radiais no galpão 
Os dispositivos Xbee foram posicionados a $30 \mathrm{~cm}$ do chão, apoiados em um suporte metálico móvel. O dispositivo conectado ao microcomputador permaneceu no início da radial enquanto o outro dispositivo percorria manualmente a radial, sendo que, os dois dispositivos estavam com a antena na vertical, mantendo a polarização coerente entre as antenas. Segundo Carthy et al. (2009), a polarização correta das antenas é um dos fatores determinantes para o bom funcionamento de sensores sem fio.

A Figura 3A mostra a foto do Xbee conectado ao computador (esquerda) e, a direita (Figura 3B), o dispositivo que percorreu as radiais.

A.

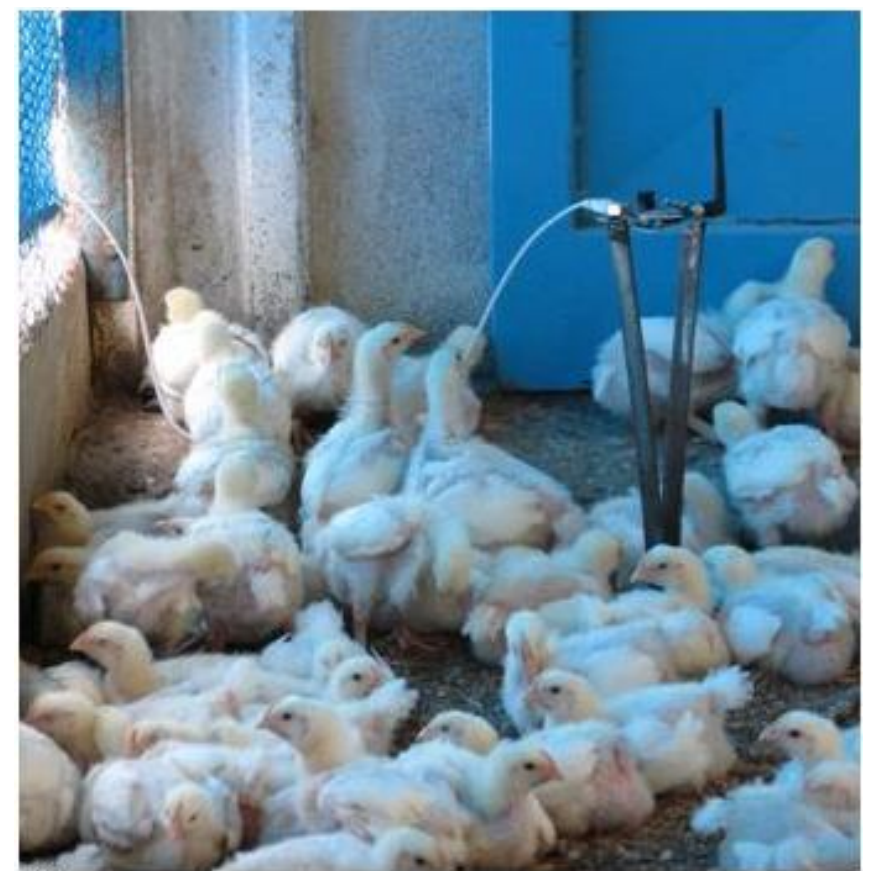

B.

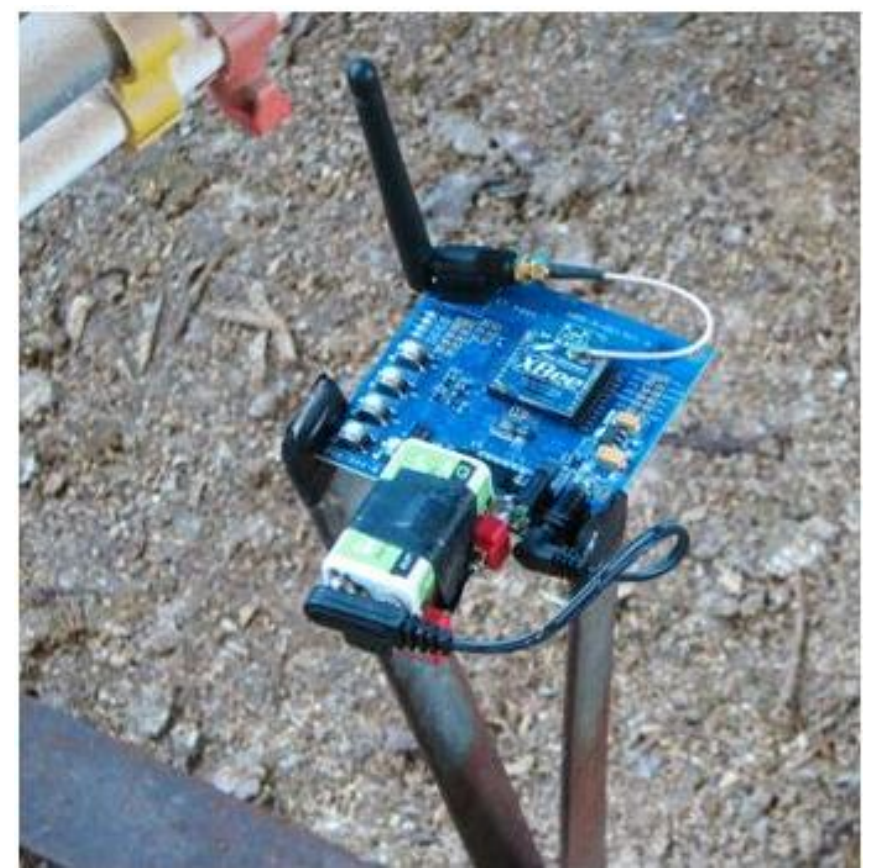

Figura 3. D ispositivos Xbee dentro da granja

\section{RESULTADOS E DISCUSSÃO}

\section{Coleta de dados de campo}

A primeira radial sobre a qual se realizaram as medidas possuía $125 \mathrm{~m}$, correspondendo ao comprimento total da granja. Na Tabela 1A estão os valores médios de potência, ruído e SNR obtidos em cada ponto.

Tabela 1. Valores médios de potência recebida, ruído detectado e razão sinal-ruído (SNR) para diferentes distâncias nas radiais

\begin{tabular}{rrrrr}
\hline \multicolumn{2}{c}{ Distância (m) } & Potência (dBm) & Ruído (dBm) & SNR (dB) \\
A. & & & & \\
& 5,00 & $-52,80$ & $-90,11$ & 37,31 \\
& 10,00 & $-55,00$ & $-90,99$ & 35,99 \\
& 15,00 & $-70,70$ & $-91,29$ & 20,59 \\
& 20,00 & $-60,33$ & $-90,14$ & 29,81 \\
& 25,00 & $-71,20$ & $-91,97$ & 20,77 \\
& 30,00 & $-66,00$ & $-91,21$ & 25,21 \\
& 40,00 & $-79,33$ & $-90,35$ & 11,02 \\
& 50,00 & $-72,00$ & $-90,45$ & 18,45 \\
& 75,00 & $-87,29$ & $-90,10$ & 2,81 \\
& 100,00 & $-83,00$ & $-90,94$ & 7,94 \\
& 125,00 & $-80,70$ & $-90,94$ & 10,24 \\
\hline B. $\quad$ & & & \\
& 2,61 & $-47,60$ & $-90,81$ & 43,21 \\
& 5,22 & $-53,60$ & $-90,45$ & 36,85 \\
& 10,44 & $-56,80$ & $-90,76$ & 33,96 \\
15,66 & $-67,33$ & $-90,44$ & 23,11 \\
& 20,88 & $-73,43$ & $-91,97$ & 18,54 \\
26,10 & $-78,50$ & $-91,38$ & 12,88 \\
& 36,54 & $-69,50$ & $-91,51$ & 22,01 \\
\hline
\end{tabular}

Obteve-se, a partir dos dados tabelados, o gráfico da Figura 4A de dispersão da SNR pela sua respectiva distância.

A curva na Figura 4A representa a curva de tendência logarítmica dos pontos medidos, através do qual se obteve um coeficiente de determinação razoável, igual a 0,80. A Eq. 2 representante desta curva é:

$$
\mathrm{SNR}[\mathrm{dB}]=-2,370 \times 10 \log (\mathrm{d})+55,250
$$

Segundo o modelo de predição Shadowing, o valor de $\beta$ é igual a 2,370, característico para a radial 1 .

Comparando-se com o espaço livre, observa-se que, devido às condições físicas (obstáculos) existentes na granja e as possíveis interferências de equipamentos elétricos, $o \beta$ da radial 1 é 1,370 maior que os $2 \mathrm{~dB}$ característicos do espaço livre.

Para a segunda bateria de medidas realizadas sobre a radial 2, obteve-se a Tabela 1B, na qual são expostos os valores obtidos, em diferentes distâncias, de potência recebida, ruído detectado e SNR médios; em seguida, os mesmos dados apresentados na Tabela 1B são apresentados em forma gráfica (Figura 4B).

A curva na Figura 4B representa a curva de tendência logarítmica dos pontos medidos na radial 2, que obteve um coeficiente de determinação razoável, igual a 0,92. A Eq. 3 representante desta curva, é:

$$
\mathrm{SNR}[\mathrm{dB}]=-2,462 \times 10 \log (\mathrm{d})+54,120
$$



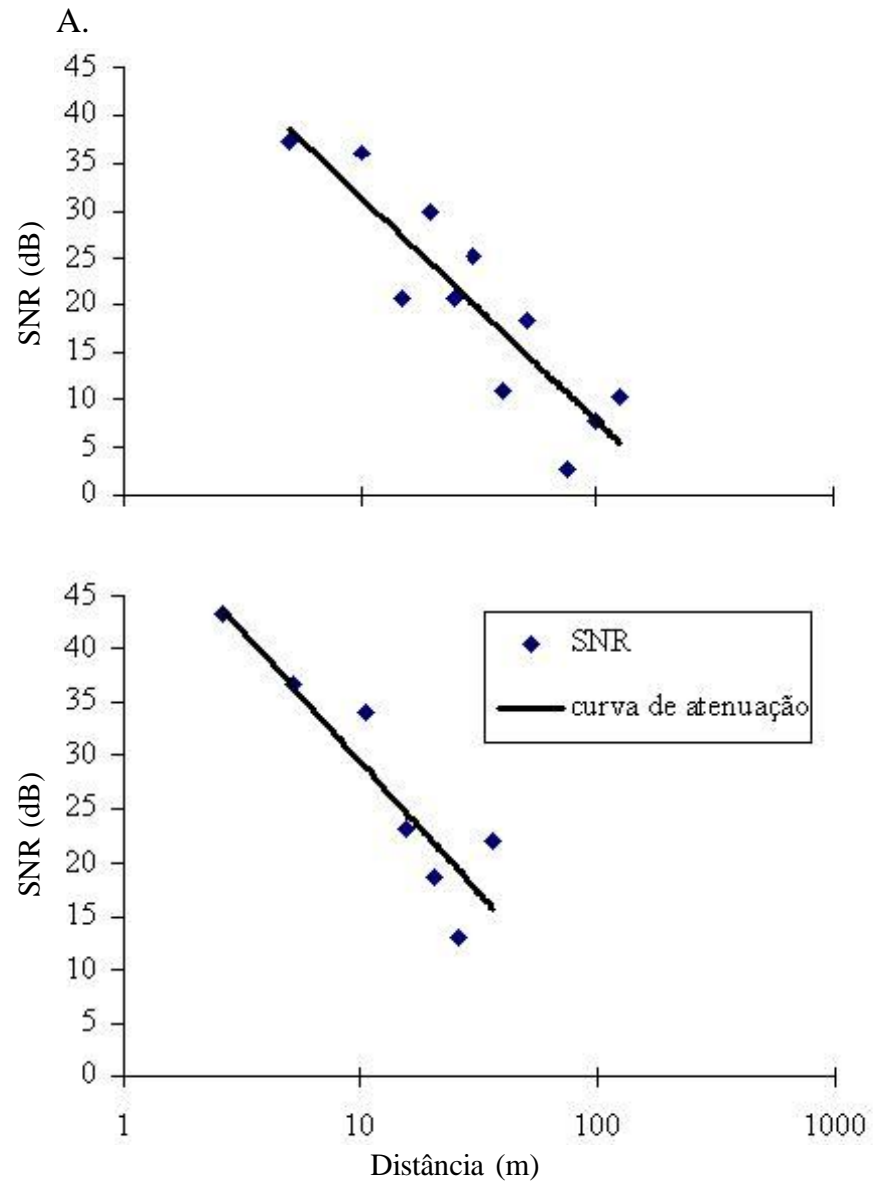

Figura 4. G ráfico de variação da razão sinal-ruído (SN R) pela distância na radiais

Novamente, segundo o modelo de predição Shadowing, o valor de $\beta$ é igual a 2,462, característico para a radial 2.

Como a radial 1 representa uma reta longitudinal do galpão e a radial 2 se refere à sua diagonal, tem-se um $\beta$ médio (Eq. 4) a partir da média aritmética dos valores obtidos nas radiais:

$$
\beta_{-} \text {médio }=(\beta 1+\beta 2) / 2
$$

Substituindo-se os valores de $\beta 1$ e $\beta 2$, tem-se (Eq. 5):

$$
\beta_{-} \text {médio }=2,416
$$

Então, o $\beta$ característico da granja é igual a 2,416, ou 1,416 $\mathrm{dB}$ maior que no espaço livre. Analogamente, o offset de atenuação médio (Eq. 6) da granja é:

$$
X \_ \text {médio }=(\mathrm{X} 1+\mathrm{X} 2) / 2
$$

Substituindo-se os valores de X1 e X2, tem-se (Eq. 7):

$$
\text { X_médio }=54,688
$$

Então, a equação característica da granja (Eq. 8) é:

$$
\operatorname{SNR}[\mathrm{dB}]=-2,416 \times 10 \log (\mathrm{d})+54,688
$$

A Figura 5 representa a curva característica da granja.



Figura 5. Curva característica da granja

\section{Análise dos resultados}

Segundo a norma IEEE 802.15.4 (2003), para o bom desempenho dos dispositivos IEEE 802.15.4 se recomenda uma taxa de erro de bit de $10^{-5}$, ou seja, um bit errado para cada cem mil bits transmitidos. Ainda de acordo com a referida norma, a taxa de erro de bit é obtida com uma SNR de aproximadamente 3,5 dB. Recorrendo à curva característica da granja (Eq. 8), para o limite de 3,5 dB de SNR, a distância máxima de transmissão é $131 \mathrm{~m}$

Analisando a distância máxima dentro do galpão, que é de 125,57 m (diagonal entre vértices opostos), e comparando-a com a distância de transmissão obtida na caracterização da granja $(131 \mathrm{~m})$, chega-se à conclusão de que, para todos os pontos do galpão, haverá condições para a comunicação entre os dispositivos da RSSF, sem necessidade de equipamentos repetidores.

A não necessidade de repetidores significa que pode ser utilizada, neste ambiente, uma arquitetura estrela na RSSF, na qual todos os dispositivos se comunicam diretamente com um concentrador. Evita-se, então, o gasto de energia necessário para a repetição de pacotes entre dispositivos. Adicionalmente, o dispositivo concentrador pode ser posicionado no centro geométrico do galpão, de modo a prover uma distância média mínima entre este e os demais dispositivos, garantindo níveis adequados de SNR em todos os enlaces de comunicação possíveis.

Morais et al. (2008) utilizaram a tecnologia Zigbee para monitoramento de parreiras em Portugal, sendo que as distâncias entre dispositivos variaram de 100 a 300 m, com potências de transmissão autoajustáveis via software.

As diferenças de distâncias obtidas neste trabalho (distância máxima entre dispositivos de $131 \mathrm{~m}$ dentro da granja) com as distâncias utilizadas por Morais et al. (2008), que são maiores, são explicadas pela diferença entre os ambientes e a atenuação que eles acarretam na propagação de sinais de radiofrequência em $2,4 \mathrm{GHz}$.

Uma granja de frangos possui uma grande densidade de objetos e aves, somada às interferências causadas por equipamentos elétricos; já em uma plantação de videiras a densidade de objetos é menor devido ao espaçamento entre 
linhas e também por não possuir equipamentos elétricos potencialmente perturbadores, demonstrando que, devido aos diversos equipamentos elétricos e uma densidade maior de objetos na utilização da tecnologia Zigbee neste ambiente da granja de frango, deve-se levar em consideração a redução das distâncias entre os dispositivos para garantir níveis adequados de SNR.

\section{ConclusõEs}

1. A ferramenta computacional desenvolvida mostrou-se eficiente para as medições de potência de sinal e de energia de canal.

2. A curva de atenuação obtida indica, quanto à perspectiva de propagação de sinais, que a solução de RSSF proposta neste trabalho pode ser aplicada em granja de frango, com êxito.

\section{LITERATURA CITADA}

Carthy, U. M.; Ayalew, G.; Butler, F.; Mcdonnell, K.; Ward, S. Impact of reader antenna polarisation, distance, inlay design, conveyor speed, tag location and orientation on the coupling of UHF RFID as applied to modified atmosphere packaged meat. Computers and Electronics in Agriculture, v.69, p.135141.2009.

Fanimokun, A.; Frolik, J. Effects of natural propagation environments on wireless sensor network coverage area. In: System theory, Proceedings of the Southeastern Symposium on, 35, 2003, Morgantown. Anais... Washington: IEEE, 2003. CD-Rom

Green, O.; Nanimi, E. S.; Vidal, V. B.; Jorgensen, R. N.; Storm, I. M. L. D.; Sorensen, C. G. Monitoring and modeling temperature variations inside silage stacks using novel wireless sensor networks. Computers and Electronics in Agriculture, v.69, p.149-157. 2009.
IEEE 802.15.4. Part 15.4: Wireless medium access control (MAC) and physical layer (PHY) specifications for low-rate wireless personal area networks (LR-WPANs). New York: IEEE, 2003. 324p.

Lima, A. M. C. Avaliação de dois sistemas de produção de frango de corte: Uma visão multidisciplinar. Campinas: UNICAMP, 2005. 122p. Tese Doutorado

Morais, R.; Fernandes, M. A.; Matos, S. G.; Serônio, C.; Ferreira, P. J. S. G.; Reis, M. J. C. S. A ZigBee multi-powered wireless acquisition device for remote sensing applications in precision viticulture. Computers and Electronics in Agriculture, v.62, p.94-106. 2008.

Moura, D. J. Ambiência na avicultura de corte. In: Silva, I. J. O. Ambiência na produção de aves em clima tropical. Piracicaba: FUNEP, 2001.p. 75-149.

Nadimi, E. S.; Sogaarda, H. T.; Bakb, T.; Oudshoorna, F. W. Zigbeebased wireless sensor networks for monitoring animal presence and pasture time in a strip of new grass. Computers and Electronics in Agriculture, v.61, p.79-87, 2008.

Riquelme, J. A. L.; Soto, F.; Suardíaz, P.; Sánchez, P.; Iborra, A.; Vera, J. A. Wireless Sensor Networks for precision horticulture in Southern Spain. Computers and Electronics in Agriculture, v.68, p.25-35. 2009.

Sevegnani, K. B. Zootecnia de precisão: Análise de imagens no estudo do comportamento de frangos de corte em estresse térmico. Revista Brasileira de Engenharia Agrícola eAmbiental, v.9, p.115-119, 2005.

Silva, A. C. S.; Arce, A. I C.; Souto, S.; Costa, E. J. X. A wireless floating base sensor network for physiological responses of livestock. Computers and Electronics in Agriculture, v.49, p.246-254, 2005.

Tinôco, I. de F. F. de. Avicultura industrial: Novos conceitos de materiais, concepções e técnicas construtivas disponíveis para galpões avícolas brasileiros. Revista Brasileira de Ciência Avícola, v.3, p.01-26, 2001.

Wang, N.; Zhang, N.; Wang, M. Wireless sensors in agriculture and food industry-Recent development and future perspective. Computers and Electronics in Agriculture, v.50, p.1-14. 2006. 